\title{
El control de la diabetes mellitus y sus complicaciones en Medellín, Colombia, 2001-2003
}

\author{
Alberto Villegas Perrasse, ${ }^{1}$ Sol Beatriz Abad, ${ }^{2}$ \\ Santiago Faciolince, ${ }^{3}$ Nayeli Hernández, ${ }^{4}$ Camilo Maya, ${ }^{5}$ \\ Lucrecia Parra, $^{3}$ Edison Rivas $^{6}$ y Pilar Vallejo
}

Forma de citar Villegas Perrasse A, Abad SB, Faciolince S, Hernández N, Maya C, Parra L, et al. El control de la diabetes mellitus y sus complicaciones en Medellín, Colombia, 2001-2003. Rev Panam Salud Publica. 2006;20(6):393-402.

RESUMEN Objetivos. Identificar las principales características sociodemográficas, clínicas y conductuales de los pacientes que participaban en los programas de atención al diabético en Medellín, Colombia, y evaluar el cumplimiento de las metas de control metabólico y de los estándares de control en el diabético.

Métodos. Se realizó un estudio descriptivo transversal mediante el análisis de las historias clínicas de todos los pacientes que asistían a los programas de atención al diabético desde al menos 6 meses en nueve instituciones de salud entre enero de 2001 y diciembre de 2003. Se tomaron datos sociodemográficos (edad y sexo), clínicos (tiempo de evolución de la enfermedad, tipo y tratamiento de la diabetes y enfermedades concurrentes y su tratamiento), sobre los hábitos de vida (práctica de ejercicios físicos, hábito de fumar y consumo de azúcar y grasas) y sobre las complicaciones crónicas y los resultados de las pruebas de laboratorio del último año. Todos los datos confusos of faltantes se registraron como ausentes.

Resultados. De las 358 historias clínicas evaluadas, se logró conocer el tipo de diabetes que padecían 3554 pacientes, de ellos 95,1\% correspondieron a pacientes con diabetes tipo 2. En general, 56,9\% (intervalo de confianza de 95\% [IC95\%]: 55,2 a 58,6\%) de los pacientes realizaban ejercicios periódicos; 15,1\% (IC95\%: 13,9 a 16,3\%) aún fumaba en el momento del corte, 17,7\% (IC95\%: 16,4 a 19,0\%) había dejado de fumar y 67,2\% (IC95\%: 65,6 a 68,8\%) nunca había fumado. En total, 21,0\% (IC95\%: 19,6 a 22,5\%) de los pacientes consumía azúcar regularmente, mientras 24,8\% (IC95\%: 23,3 a 26,3\%) consumía grasas y solo 19,5\% (IC95\%: 17,8 a $21,3 \%$ ) realizaba automonitoreo de la glucemia con una frecuencia semanal o mayor. La prevalencia general de hipertensión arterial fue de 68,8\% y de 98,2\% de dislipidemia en los diabéticos tipo 2. El promedio general del índice de masa corporal fue de 28,0 kg/m² (IC95\%: 27,8 a 28,2). El promedio del número de medicamentos utilizados fue de 1,3 para el tratamiento de la diabetes tipo 2, de 1,9 para la hipertensión arterial y de 0,6 para la dislipidemia. No se encon-

Servicio de Endocrinología, Hospital Universitario San Vicente de Paul, Universidad de Antioquia, Antioquia, Colombia. La correspondencia se debe dirigir a Alberto Villegas Perrasse, Servicio de Endocrinología, Universidad de Antioquia, Hospital Universitario San Vicente de Paul, calle 64 carrera 51D, Medellín, Antioquia, Colombia. Correo electrónico: perrasse70@epm.net.co
2 Empresa Promotora de Salud Punto Salud Susalud, Medellín, Colombia.

3 Institución Prestadora de Salud Comfama, Medellín, Colombia.

4 Institución Prestadora de Salud Copsana, Medellín, Colombia.
5 Empresa Social del Estado Metro Salud, Medellín, Colombia.

6 Institución Prestadora de Salud Comfenalco Cajaser, Medellín, Colombia.

7 Coomeva Medicina Prepagada, Medellín, Colombia. 
traron los datos de dislipidemia de colesterol de lipoproteínas de baja densidad ( $L D L$ ) en 44,8\% de las historias clinicas y de colesterol de lipoproteínas de alta densidad (HDL) en 16,4\%. Las complicaciones oculares fueron las más frecuentes (31,8\%; IC95\%: 30,1 a 33,5\%), seguidas de las afecciones renales (25,9\%; IC95\%: 24,4 a 27,5\%) y las cardiovasculares (22,5\%; IC95\%: 21,1 a 23,8\%). El cumplimiento de las metas de control del colesterol de LDL, los triglicéridos y el colesterol de HDL fue insuficiente (14,2\%, 36,9\% y 47,4\%, respectivamente).

Conclusiones. En la población estudiada, las tres complicaciones crónicas que más influyen en el pronóstico y el costo de salud en los diabéticos fueron la nefropatía, la retinopatía y la dislipidemia. El gran subregistro de datos encontrado en las historias clínicas se puede reducir si se toman medidas para estandarizar las historias clinicas y se realizan evaluaciones periódicas de la calidad de las mismas.

Palabras clave Diabetes mellitus, complicaciones de la diabetes, Colombia.
La diabetes es una enfermedad provocada por la alteración del metabolismo de los carbohidratos, grasas y proteínas y produce complicaciones en diferentes órganos (1). El tratamiento de la diabetes mellitus requiere un enfoque interdisciplinario, ya que comprende numerosos aspectos farmacológicos, nutricionales, educacionales, sociales y psicológicos $(1,2)$. Se calcula que la prevalencia de diabetes mellitus en América Latina aumentará en 250\% en los próximos 20 años $(2,3)$, con lo que se incrementará también la frecuencia de sus complicaciones, como la ceguera, la insuficiencia renal crónica y el pie diabético. Además, la diabetes mellitus está reconocida como un equivalente cardiovascular debido a su asociación con la enfermedad coronaria $(4,5)$. Los pacientes diabéticos consumen $11 \%$ del presupuesto total de salud en los Estados Unidos de América y alrededor de $6 \%$ del costo en salud en América Latina y el Caribe (6-8).

En los últimos años, los mayores esfuerzos en la lucha contra la diabetes se han enfocado hacia la prevención y el tratamiento de las complicaciones crónicas. El Estudio sobre el Control y las Complicaciones de la Diabetes, realizado en pacientes con diabetes tipo 1 (9), el Estudio sobre el Control y las Complicaciones de la Diabetes en el Reino Unido, en pacientes con diabetes tipo 2 (10), y el Estudio sobre la Prevención de las Complicaciones Mediante Terapia Intensiva con Insulina en Diabéticos Tipo 2, realizado en $\mathrm{Ku}$ mamoto, Japón (11), demostraron que el tratamiento intensivo de la diabetes retarda el inicio de las complicaciones microvasculares.

Para poder aplicar intervenciones adecuadas y oportunas es necesario conocer las características de la enfermedad y de sus complicaciones crónicas más frecuentes. Sin embargo, poco se sabe acerca de las principales complicaciones crónicas de la diabetes mellitus, su prevalencia y su evolución en Colombia $(12,13)$.

El objetivo del presente estudio fue identificar las principales características sociodemográficas, clínicas y conductuales de los pacientes que participaban en los programas de atención al diabético en Medellín, Colombia, y evaluar el cumplimiento de las metas de control metabólico y de los estándares de control en los pacientes diabéticos.

\section{MATERIALES Y MÉTODOS}

Se realizó un estudio descriptivo transversal mediante el análisis de las historias clínicas de todos los pacientes que asistían a los programas de atención al diabético desde al menos 6 meses en nueve instituciones prestadoras de servicios de salud (IPS) y empresas promotoras de salud (EPS) de la ciudad de Medellín, Colombia. La investigación se realizó entre enero de 2001 y diciembre de 2003 y en ella participaron pacientes de: Coomeva EPS Conquistadores $(n=166 ; 4,6 \%$ del total de participantes), Coomeva EPS Medicina Prepagada $(n=94 ; 2,6 \%)$, EPS
Punto Salud Susalud ( $n=811 ; 22,6 \%$ ), IPS Comfama ( $n=1611 ; 45,0 \%)$, IPS Comfenalco Cajaser $(n=120 ; 3,3 \%)$, IPS Copsana $(n=89 ; 2,5 \%)$, Instituto del Tórax (IPS) $(n=290 ; 8,1 \%)$, Servicio de Salud de la Universidad Nacional (IPS) $(n=99 ; 2,8 \%)$ y el Centro de Atención Ambulatoria Central del Instituto de los Seguros Sociales (ISS) Seccional Antioquia (EPS) $(n=303 ; 8,5 \%)$. En total participaron 3583 pacientes, todos adultos, ya que los menores se atienden en los servicios de pediatría.

Los datos sociodemográficos (edad y sexo) y clínicos (tiempo de evolución de la enfermedad, tipo y tratamiento de la diabetes, enfermedades concurrentes -hipertensión, dislipidemia y obesidad-y su tratamiento), así como la información sobre los hábitos de vida (práctica de ejercicios físicos, hábito de fumar y consumo de azúcar y grasas), las complicaciones crónicas y los resultados de las pruebas de laboratorio del último año se colectaron a partir de las historias clínicas mediante un formulario estructurado. Todos los datos confusos o faltantes se registraron como ausentes. Se evaluó el llenado adecuado de las historias clínicas - uno de los objetivos de esta investigación- de acuerdo con la cantidad de datos ausentes en las historias.

Se consideró que el paciente presentaba hipertensión arterial (HTA) cuando en dos mediciones o más la tensión arterial fue mayor que 140/80 mm Hg o si seguía un tratamiento contra la HTA. Se estableció el diagnóstico de dislipidemia cuando se presentaba 
alguna de las siguientes condiciones: colesterol de lipoproteínas de baja densidad (LDL) $\geq 100$, triglicéridos $\geq 150$, colesterol de lipoproteínas de alta densidad (HDL) $<40$, colesterol total $\geq 180$ o si el paciente se encontraba en tratamiento por dislipidemia. Los datos de LDL se tomaron de las historias clínicas y además se calcularon a partir de los últimos datos de colesterol total, colesterol de HDL y triglicéridos encontrados en las historias, excepto cuando los triglicéridos eran mayores que $400 \mathrm{mg} / \mathrm{dL}$. Para la evaluación de la HTA y de la dislipidemia se tomaron en cuenta los datos del último control anotado en la historia y si se presentaban dudas se revisaban los datos del último año.

Se tomaron los datos de la evaluación oftalmológica si esta se había realizado en el último año y contenía la información del evaluador. Se registraron las complicaciones y hábitos de vida recogidos en las historias clínicas durante el período de evolución. Se consideró que el paciente hacía ejercicios físicos periódicos si según su última evaluación había realizado por lo menos 30 minutos de ejercicios al menos tres veces a la semana por un mínimo de 3 meses consecutivos.

El grado de control de los diferentes parámetros estudiados se determinó según los estándares publicados por la Asociación Estadounidense de Diabe- tes y la Asociación Latinoamericana de Diabetes $(2,14)$.

La recolección de datos estuvo a cargo de médicos generales, médicos especialistas en diabetes y estudiantes de medicina que recibieron entrenamiento, tanto en la recolección de datos para la investigación como en los posibles problemas que se podían presentar. Se comprobó la reproducibilidad entre los encuestadores mediante ejercicios de prueba con historias clínicas. La información se digitó y procesó en una base de datos en MS Access (Microsoft Corporation, Redmond, WA, Estados Unidos). Para el análisis descriptivo de las variables se obtuvieron medidas de tendencia central y de dispersión, tales como la media, la frecuencia, la desviación estándar (DE) y los intervalos de confianza de 95\% (IC95\%).

La investigación se realizó con la autorización previa de los directores de las instituciones participantes. Por tratarse de un estudio descriptivo retrospectivo, en ningún momento hubo intervención en el tratamiento de los pacientes.

\section{RESULTADOS}

En 3554 de las 3583 historias clínicas evaluadas estaba registrado el tipo de diabetes que padecía el paciente, de ellas $95,1 \%$ correspondieron a pacien- tes con diabetes tipo 2. La edad promedio de la población estudiada fue de 59,8 años (IC95\%: 59,4 a 60,3). La relación entre mujeres y hombres fue de $1,4: 1$; no se observaron diferencias estadísticamente significativas entre los pacientes con diabetes tipos 1 y 2 en cuanto al sexo $(P=0,780)$. El tiempo transcurrido desde el diagnóstico de la diabetes hasta el momento del corte en la población estudiada fue de 8,4 años (IC95\%: 8,2 a 8,7), mayor en los pacientes con diabetes tipo 1 (cuadro 1 ). La edad promedio al momento del diagnóstico fue de 51,3 años (IC95\%: 50,8 a 51,8), menor en los casos de diabetes tipo 1 (22,7 años; IC95\%: 20,1 a $25,3)$ que en los del tipo 2 (52,5 años; IC95\%: 52,1 a 52,9) $(P<0,0001)$.

Según la información sobre los hábitos de salud, 56,9\% (IC95\%: 55,2 a $58,6 \%$ ) de los pacientes realizaban ejercicios periódicamente; 15,1\% (IC95\%: 13,9 a $16,3 \%$ ) aún fumaba en el momento del corte, 17,7\% (IC95\%: 16,4 a $19,0 \%$ ) había dejado de fumar y $67,2 \%$ (IC95\%: 65,6 a 68,8\%) nunca había fumado. En total, 21,0\% (IC95\%: 19,6 a $22,5 \%$ ) de los pacientes consumía azúcar regularmente, mientras 24,8\% (IC95\%: 23,3 a 26,3\%) consumía grasas y solo $19,5 \%$ (IC95\%: 17,8 a 21,3\%) realizaba automonitoreo de la glucemia con una frecuencia semanal o mayor.

Al evaluar las enfermedades asociadas con la diabetes se encontró que

CUADRO 1. Datos generales de la población estudiada, según el tipo de diabetes ${ }^{a}$

\begin{tabular}{|c|c|c|c|c|c|c|c|c|}
\hline Variable & $\begin{array}{c}\text { Pacientes } \\
\text { con } \\
\text { diabetes } \\
\text { tipo } 1\end{array}$ & $\mathrm{DE}(\mathrm{IC} 95 \%)^{\mathrm{b}}$ & $\begin{array}{c}\text { Pacientes } \\
\text { con } \\
\text { diabetes } \\
\text { tipo } 2\end{array}$ & DE (IC95\%) & $\begin{array}{c}\text { Tipo de } \\
\text { diabetes } \\
\text { desconocida }\end{array}$ & DE (IC95\%) & Total & DE (IC95\%) \\
\hline Número (\%) & $145(4,0)$ & $N A^{c}$ & $3409(95,1)$ & NA & $29(0,8)$ & NA & 3583 & NA \\
\hline Mujeres, \% & 57,2 & NA & 58,4 & NA & 58,6 & NA & 58,4 & NA \\
\hline Hombres, \% & 42,8 & NA & 41,5 & NA & 41,4 & NA & 41,6 & NA \\
\hline $\begin{array}{l}\text { Edad, años } \\
\text { Edad al momento }\end{array}$ & 33,7 & $5,6(31,2-36,2)$ & 60,9 & $7,8(60,5-61,4)$ & 61,0 & $7,0(55,2-66,7)$ & 59,8 & $7,7(59,4-60,3)$ \\
\hline $\begin{array}{l}\text { del diagnóstico, años } \\
\text { Tiempo desde }\end{array}$ & 22,7 & $15,2(20,1-25,3)$ & 52,5 & $12,4(52,1-52,9)$ & $50,0^{e}$ & $23,5(23,2-77,0)^{\mathrm{e}}$ & 51,3 & $7,1(50,8-51,8)$ \\
\hline el diagnóstico, años & 11,1 & $3,1(9,6-12,7)$ & 8,3 & $2,8(8,0-8,6)$ & 7,0 & $9,6(4,1-18,1)^{d}$ & 8,4 & $2,9(8,2-8,7)$ \\
\hline
\end{tabular}

a El número total de historias clínicas analizadas fue de 3 583. Los porcentajes están calculados a partir del número total de expedientes que contenían el dato analizado.

b DE: desviación estándar; IC95\%: intervalo de confianza de 95\%.

cNA: no se aplica.

d Solo se pudo encontrar el dato de tiempo de diagnóstico en 3 de los 29 pacientes.

e Basado en $n=3$. 
CUADRO 2. Frecuencia de las principales enfermedades asociadas con la diabetes en la población estudiada, según el tipo de diabetes

\begin{tabular}{|c|c|c|c|c|}
\hline \multirow{2}{*}{$\begin{array}{c}\text { Enfermedades } \\
\text { asociadas }\end{array}$} & $\begin{array}{l}\text { Diabetes } \\
\text { tipo } 1\end{array}$ & $\begin{array}{c}\text { Diabetes } \\
\text { tipo } 2 \\
\end{array}$ & Total & \multirow{2}{*}{$\begin{array}{c}\text { Subdiagnóstico } \\
\text { de la } \\
\text { comorbilidad }^{\mathrm{b}}\end{array}$} \\
\hline & $\%(I C 95 \%)^{a}$ & $\%(I C 95 \%)$ & $\%(I C 95 \%)$ & \\
\hline Hipertensión arterial & $23,2(16,3-30,2)$ & $71,1(69,5-72,6)$ & $68,8(66,7-69,8)$ & $28(0,8 \%)$ \\
\hline Dislipidemia & $40,9(31,4-48,6)$ & $98,4(98,0-98,8)$ & $98,4(97,9-98,8)$ & 1177 (34,2\%) \\
\hline Sobrepeso & $24,2(17,2-32,5)$ & $44,1(42,4-45,9)$ & $43,1(41,4-44,9)$ & NA \\
\hline Obesidad & $12,1(7,1-18,9)$ & $30,2(28,6-31,8)$ & $29,5(28,0-31,2)$ & $N A^{c}$ \\
\hline
\end{tabular}

a IC95\%: intervalo de confianza de 95\%.

b Subdiagnóstico: número de pacientes (\%) con hipertensión arterial y dislipidimia según los resultados de las pruebas clínicas que aparecían en sus historias clínicas, pero sin el diagnóstico explícito.

${ }^{c}$ NA: No se aplica.
68,8\% (IC95\%: 66,7 a 69,8\%) del total de pacientes presentaba HTA, algo superior en los casos con diabetes tipo 2 (71,1\%; IC95\%: 69,5 a 72,6\%). Solo 67,9\% (IC95\%: 67,6 a 70,9\%) de los diabéticos tipo 2 tenían diagnóstico de dislipidemia en sus historias clínicas, sin embargo, $34,2 \%$ de los pacientes sin ese diagnóstico presentaban dislipidemia según los cálculos realizados a partir de los valores de colesterol y triglicéridos, lo que resultó en una proporción total de 98,4\% (IC95\%: 97,9 a $98,8 \%$ ) (cuadro 2).

El promedio general del índice de masa corporal (IMC) fue de $28,0 \mathrm{~kg} / \mathrm{m}^{2}$ (DE = 5,3; IC95\%: 27,8 a 28,2), menor en los casos con diabetes tipo 1 $\left(24,5 \mathrm{~kg} / \mathrm{m}^{2} ; \mathrm{DE}=4,8 ;\right.$ IC95\%: $23,5 \mathrm{a}$ $25,4)$ que en los de tipo $2\left(28,2 \mathrm{~kg} / \mathrm{m}^{2}\right.$; $\mathrm{DE}=5,3$; IC95\%: 28,0 a 28,4). En general, 74,3\% (IC95\%: 72,7 a 75,8\%) de los diabéticos tipo 2 tenían IMC $>25 \mathrm{~kg} / \mathrm{m}^{2}$, mientras que solo $36,3 \%$ (IC95\%: 28,2 a 45,2\%) de los diabéticos tipo 1 estaban en esa categoría.

\section{Tratamiento de la diabetes y de las enfermedades relacionadas}

En cuanto al tratamiento, 11,7\% (IC95\%: 7,0 a 18,1\%) de los pacientes con diabetes tipo 1 utilizaba insulina una vez al día, 73,0\% (IC95\%: 65,1 a $80,1 \%$ ) la utilizaba dos veces al día y 13,0\% (IC95\%: 3,4 a 12,3\%) lo hacía tres veces al día; dos pacientes utilizaban metformina como parte del trata- miento. El promedio del número de medicamentos utilizados para el tratamiento de la diabetes tipo 2 fue de 1,3; 44,2\% (IC95\%: 42,4 a 46,0\%) de los pacientes utilizaba un medicamento, 36,2\% (IC95\%: 34,5 a 38,0\%) utilizaba dos, 3,8\% (IC 95\% de 3,1 a 4,5\%) empleaba tres y dos pacientes $(0,1 \%$; IC95\%: 0,0 a 0,2\%) utilizaban cuatro medicamentos. La combinación más frecuentemente utilizada fue la de sulfonilúreas y metformina $(27,3 \%$; IC95\%: 25,9 a 28,9\%), seguida de metformina con insulina (6,0\%; IC95\%: 5,3 a $6,9 \%)$; solo $6(0,2 \%)$ pacientes utilizaban tiazolidinedionas y ninguno utilizaba metiglinidas o acarbosa. En el cuadro 3 se muestran los principales tratamientos utilizados por los pacientes estudiados con diabetes tipo 2.
Para el tratamiento de la HTA se empleaban en promedio 1,9 medicamentos por paciente: 2,9\% (IC95\%: 2,2 a 3,5\%) de los pacientes no utilizaba tratamientos farmacológicos, mientras que 38,9\% (IC95\%: 37,0 a 40,9\%) utilizaba un medicamento, 34,5\% (IC95\%: 32,6 a $36,4 \%$ ) dos medicamentos, $18,4 \%$ (IC95\%: 16,8 a 19,9\%) tres medicamentos y 5,3\% (IC95\%: 4,0 a 5,7\%) cuatro medicamentos o más. Los inhibidores de la enzima convertidora de la angiotensina (IECA) fueron los medicamentos prescritos con mayor frecuencia (72,1\%; IC95\%: 70,3 a 73,9\%), seguidos por los diuréticos (42,6\%; IC95\%: 40,7 a 44,6\%), los antagonistas del calcio (36,1\%; IC95\%: 34,2 a 38,0\%), los $\beta$-bloqueadores (15,3\%; IC95\%: $13,8$ a $16,7 \%)$, los $\alpha$-antagonistas (5,5\%; IC95\%: 4,6 a 6,4\%) y otros medicamentos (13,3\%; IC95\%: 11,9 a 14,6\%). En el cuadro 4 se presentan los medicamentos y sus combinaciones más frecuentes.

El promedio de medicamentos utilizados por los pacientes para el tratamiento de la dislipidemia fue de 0,6; 50,0\% (IC95\%: 47,8 a 52,6\%) de los pacientes no tenía tratamiento farmacológico, 47,4\% (IC95\%: 44,9 a 49,8\%) utilizaba un medicamento, 4,7\% (IC95\%: 1,4 a $8,0 \%$ ) tomaba dos medicamentos y $0,2 \%$ (IC $95 \%$ : 0,0-0,3\%) tomaba tres. La lovastatina fue el medicamento más frecuentemente utilizado (30,1\%; IC95\%: 28,0 a 31,1\%), seguido de los fibratos (19,1\%; IC95\%: 17,8 a
CUADRO 3. Medicamentos utilizados para el tratamiento de la diabetes tipo 2 en la población estudiada

\begin{tabular}{lcc}
\hline \multicolumn{1}{c}{ Medicamentos } & $\begin{array}{c}\text { Pacientes que utilizan } \\
\text { este medicamento, } \%\end{array}$ & IC95\%a \\
\hline Sin tratamiento farmacológico & 15,5 & $14,2-16,8$ \\
Sulfonilúreas & 58,2 & $56,5-59,8$ \\
Metformina & 48,2 & $46,7-50,0$ \\
Sulfonilúreas + metformina & 27,3 & $25,9-28,9$ \\
Insulina & 19,6 & $18,5-21,2$ \\
Metformina + insulina & 6,0 & $5,3-6,9$ \\
Insulina una vez al día & 7,5 & $6,7-8,5$ \\
Insulina dos veces al día & 11,6 & $10,5-12,7$ \\
Insulina tres veces al día & 0,7 & $0,5-1,1$ \\
Tiazolidinendionas & 0,2 & $0,0-0,3$ \\
\end{tabular}

a IC95\%: intervalo de confianza de 95\%. 
CUADRO 4. Principales medicamentos antihipertensivos utilizados en la población estudiada

\begin{tabular}{lcc}
\hline \multicolumn{1}{c}{ Medicamentos } & $\begin{array}{c}\text { Pacientes que utilizan } \\
\text { este medicamento, \% }\end{array}$ & IC95\%a \\
\hline IECA $^{\text {b }}$ & 17,9 & $16,4-19,1$ \\
Diuréticos, IECA & 7,8 & $6,9-8,7$ \\
IECA, antagonistas del calcio & 5,1 & $4,4-5,8$ \\
Diuréticos, IECA, antagonistas del calcio & 4,6 & $3,9-5,3$ \\
Antagonistas del calcio & 4,5 & $3,8-5,2$ \\
Diuréticos, antagonistas del calcio & 3,7 & $3,1-4,4$ \\
Diuréticos, IECA, $\beta$-bloqueadores & 2,4 & $1,9-2,9$ \\
Otras combinaciones & 26,4 & $24,9-27,8$ \\
Sin tratamiento farmacológico & 2,9 & $2,2-3,5$ \\
\hline & &
\end{tabular}

$20,5 \%)$ y la combinación de fibratos y lovastatina (1,9\%; IC95\%: 1,5 a 2,5\%).

\section{Control metabólico y calidad de la atención}

La proporción de pacientes que alcanzaron las metas de control metabólico según el tipo de diabetes se presenta en el cuadro 5. En los pacientes con diabetes tipo 1 se observó un mayor control de todas las metas, excepto la del control de la diabetes. La dislipidemia fue el parámetro menos controlado, ya que solo $15,9 \%$ de los pacientes con diabetes tipo 2 tenían niveles de colesterol de LDL por debajo de $100 \mathrm{mg} / \mathrm{dL}$.
En general, $42,8 \%$ del total de la población estudiada $(26,2 \%$ de los casos con diabetes tipo 1 y $43,6 \%$ de los casos con el tipo 2) presentaron valores de glucohemoglobina por debajo de 7,0\%. El IMC fue menor de $25 \mathrm{~kg} / \mathrm{m}^{2}$ en $27,0 \%$ del total de casos $(62,1 \%$ de los pacientes con diabetes tipo 1 y $25,5 \%$ de los de tipo 2). El control de la tensión arterial sistólica por debajo de $140 \mathrm{~mm} \mathrm{Hg}$ se observó en $46,3 \%$ del total de la población $(75,2 \%$ de los pacientes con diabetes tipo $1 \mathrm{y}$ $44,8 \%$ de los de tipo 2), mientras que la tensión arterial diastólica estuvo por debajo de $80 \mathrm{~mm} \mathrm{Hg}$ en $36,8 \%$ de los pacientes $(57,9 \%$ de los casos con diabetes tipo 1 y $35,9 \%$ de los de tipo 2) (cuadro 5).
Se observó una elevada proporción de datos ausentes, muchos de ellos necesarios para la evaluación del diagnóstico precoz de las complicaciones, como los de microalbuminuria en $72,9 \%$ de los pacientes y de la evaluación oftalmológica en 53,6\% (cuadro 6). A pesar de que el nivel de colesterol de LDL es un parámetro fundamental para seguir la evolución y el tratamiento de la dislipidemia, no se encontró este dato en $44,8 \%$ de las historias clínicas analizadas. La ausencia de los demás parámetros varió entre $0,6 \%$ (tensión arterial) y 16,4\% (colesterol de HDL).

\section{Complicaciones vasculares}

Para la evaluación de las complicaciones micro y macrovasculares se utilizó el diagnóstico clínico registrado en la historia o los resultados de los exámenes de laboratorio que permitieran llegar a un diagnóstico. Un gran número de las historias analizadas no tenían los datos de laboratorio, por lo que la frecuencia de las complicaciones encontradas no refleja la situación real en esta población (cuadro 7). De acuerdo con los datos disponibles, las complicaciones oculares fueron las más frecuentes (31,8\%; IC95\%: 30,1 a $33,5 \%)$, seguidas de las afecciones renales (25,9\%; IC95\%: 24,4 a 27,5\%) y las cardiovasculares (22,5\%; IC $95 \%$ : 21,1 a $23,8 \%$ ). No se encontraron pacientes con transplantes renales y solo 7

CUADRO 5. Porcentajes de cumplimiento de las metas de control en la población estudiada, según el tipo de diabetes

\begin{tabular}{|c|c|c|c|c|c|c|c|c|}
\hline \multirow[b]{2}{*}{ Metas } & \multirow[b]{2}{*}{ Característica } & \multicolumn{2}{|c|}{$\begin{array}{c}\text { Diabéticos tipo } 1 \\
\quad(n=145)\end{array}$} & \multicolumn{2}{|c|}{$\begin{array}{c}\text { Diabéticos tipo } 2 \\
(n=3409)\end{array}$} & \multicolumn{2}{|c|}{$\begin{array}{c}\text { Total }^{\mathrm{a}} \\
(n=3583)\end{array}$} & \multirow{2}{*}{$\begin{array}{l}\text { Valor límite } \\
\text { esperado }\end{array}$} \\
\hline & & $\%$ & IC95\%b & $\%$ & IC95\% & $\%$ & IC95\% & \\
\hline \multirow[t]{6}{*}{ De laboratorio } & Triglicéridos & 56,6 & $48,5-64,6$ & 36,1 & $34,5-37,7$ & 36,9 & $35,3-38,4$ & $<150 \mathrm{mg} \%(1,7 \mathrm{mmol} / \mathrm{L})$ \\
\hline & Colesterol total & 37,9 & $30,0-45,8$ & 27,8 & $26,3-29,3$ & 28,2 & $26,7-29,6$ & $<180 \mathrm{mg} \%(4,65 \mathrm{mmol} / \mathrm{L})$ \\
\hline & Colesterol de HDL & 50,3 & $42,2-58,5$ & 47,3 & $45,6-49,0$ & 47,4 & $45,7-49,0$ & $>40 \mathrm{mg} \%(1,03 \mathrm{mmol} / \mathrm{L})$ \\
\hline & LDL (calculado + historia) & 21,4 & $14,7-28,1$ & 13,8 & $12,7-15,0$ & 14,2 & $13,1-15,3$ & $<100 \mathrm{mg} \%(2,6 \mathrm{mmol} / \mathrm{L})$ \\
\hline & Glucemia & 22,8 & $15,9-29,6$ & 24,3 & $22,8-25,7$ & 24,2 & $22,8-25,6$ & $<110 \mathrm{mg} \%(6,11 \mathrm{mmol} / \mathrm{L})$ \\
\hline & Glucohemoglobina & 26,2 & $19,0-33,4$ & 43,6 & $41,9-45,3$ & 42,8 & $41,2-44,5$ & $\leq 7 \%$ \\
\hline \multirow[t]{3}{*}{ Clínicas } & Tensión arterial sistólica & 75,2 & $68,1-82,2$ & 44,8 & $43,1-46,5$ & 46,3 & $44,7-47,9$ & $\leq 140 \mathrm{~mm} \mathrm{Hg}$ \\
\hline & Tensión arterial diastólica & 57,9 & $49,9-66,0$ & 35,9 & $34,3-37,5$ & 36,8 & $35,2-38,4$ & $\leq 80 \mathrm{~mm} \mathrm{Hg}$ \\
\hline & Peso corporal normal & 62,1 & $54,2-70,0$ & 25,5 & $24,1-27,3$ & 27,0 & $25,6-28,5$ & $<25 \mathrm{~kg} / \mathrm{m}^{2}$ \\
\hline
\end{tabular}

a En los totales se tomaron en cuenta los datos de los 29 pacientes de los que no se logró conocer el tipo de diabetes.

b IC95\%: intervalo de confianza de $95 \%$. 
CUADRO 6. Calidad de la atención en la población estudiada, según el tipo de diabetes

\begin{tabular}{|c|c|c|c|}
\hline \multirow[b]{3}{*}{ Característica } & \multicolumn{3}{|c|}{ Datos omitidos en la historia } \\
\hline & Diabetes tipo 1 & Diabetes tipo 2 & Total \\
\hline & $\%(\operatorname{IC95\% })^{\mathrm{a}}$ & $\%(I C 95 \%)$ & $\%(\mathrm{IC} 95 \%)$ \\
\hline Glucemia & $19,3(12,9-25,7)$ & $7,2(6,3-8,1)$ & $7,8(7,0-8,8)$ \\
\hline Glucohemoglobina & $10,3(5,4-15,3)$ & $10,1(9,1-11,1)$ & $10,2(9,2-11,2)$ \\
\hline Triglicéridos & $17,9(11,7-24,2)$ & $8,2(7,3-9,1)$ & $8,6(7,7-9,6)$ \\
\hline Colesterol total & $17,2(11,1-23,4)$ & $5,7(5,0-6,5)$ & $6,3(5,5-7,1)$ \\
\hline Colesterol de HDL & $29,6(22,2-37,1)$ & $15,7(14,5-16,9)$ & $16,4(15,2-17,6)$ \\
\hline Colesterol de LDL & $52,4(44,3-60,5)$ & $44,4(42,8-46,1)$ & $44,8(43,1-46,4)$ \\
\hline Tensión arterial sistólica & $1,3(-0,5-3,3)$ & 0 & $0,6(0,4-0,9)$ \\
\hline Tensión arterial diastólica & $1,3(-0,5-3,3)$ & 0 & $0,6(0,4-0,9)$ \\
\hline Creatinina & $22,7(15,9-29,6)$ & $11,8(10,7-12,9)$ & $12,3(11,2-13,4)$ \\
\hline Microalbuminuria & $69,6(62,2-77,1)$ & $72,9(71,5-74,5)$ & $72,9(71,5-74,4)$ \\
\hline Evaluación oftalmológica & $51,2(43,1-59,4)$ & $61,8(60,2-63,5)$ & $53,6(51,9-55,2)$ \\
\hline
\end{tabular}

a IC95\%: intervalo de confianza de $95 \%$.

(0,3\%; IC95\%: 0,1 a $0,5 \%)$ pacientes con diabetes tipo 2 se encontraban en tratamiento de reemplazo renal. No se observaron complicaciones cardiovasculares o amputación de miembros inferiores entre los pacientes con diabetes tipo 1 y entre las complicaciones neuropáticas, la impotencia fue poco frecuente, aunque esto pudo deberse a un subregistro de los casos.

\section{DISCUSIÓN}

La prevención y la promoción de la salud constituyen elementos fundamentales en el nuevo sistema de salud de Colombia, por lo que está aumentando el número de grupos de salud que atienden a la población de diabéticos. Los estándares aceptados recomiendan llevar un control estricto de la diabetes y de los factores de riesgo cardiovasculares y realizar la evaluación precoz de las complicaciones para aplicar medidas terapéuticas oportunas (14). Está demostrado que el control estricto de la diabetes permite disminuir sus complicaciones (9-11).

Los programas de control de enfermedades crónicas deben contar con mecanismos de retroalimentación ba-

CUADRO 7. Frecuencia de las principales complicaciones en la población estudiada, según el tipo de diabetes

\begin{tabular}{|c|c|c|c|c|c|c|c|c|c|}
\hline \multirow[b]{2}{*}{ Complicaciones } & \multicolumn{3}{|c|}{$\begin{array}{l}\text { Diabetes tipo1 } \\
\quad(n=145)\end{array}$} & \multicolumn{3}{|c|}{$\begin{array}{c}\text { Diabetes tipo } 2 \\
(n=3409)\end{array}$} & \multicolumn{3}{|c|}{$\begin{array}{c}\text { Total }^{\mathrm{a}} \\
(n=3583)\end{array}$} \\
\hline & $f / n^{b}$ & $\%$ & IC95\%c & $f / n$ & $\%$ & IC95\% & $f / n$ & $\%$ & IC95\% \\
\hline Retinopatía no proliferativa & 24 & 22,2 & $14,4-30,1$ & 441 & 19,6 & $17,9-21,2$ & 468 & 19,7 & $18,1-21,3$ \\
\hline Retinopatía proliferativa & 4 & 3,7 & $0,1-7,3$ & 123 & 5,5 & $4,6-6,5$ & 127 & 5,4 & $4,5-6,3$ \\
\hline Ceguera por diabetes & 3 & 2,7 & $-0,3-5,8$ & 22 & 0,9 & $0,5-1,3$ & 25 & 1,0 & $0,6-1,3$ \\
\hline Nefropatía avanzada & 12 & 13,0 & $6,2-19,9$ & 401 & 17,0 & $15,5-18,5$ & 414 & 16,7 & $15,3-18,2$ \\
\hline Neurológicas & $10 / 123$ & 8,1 & $3,3-13,0$ & 499/2 948 & 16,9 & $15,6-18,3$ & $512 / 3094$ & 16,5 & $15,2-17,9$ \\
\hline Neuropatía periférica & 10 & 10,8 & $4,5-17,0$ & 312 & 12,9 & $11,6-14,2$ & 324 & 12,8 & $11,5-14,1$ \\
\hline Impotencia & 2 & 1,4 & $-0,5-3,3$ & 193 & 5,7 & $4,9-6,4$ & 197 & 5,5 & $4,8-6,2$ \\
\hline Pie diabético & $2 / 133$ & 1,5 & $-0,6-3,6$ & $188 / 3138$ & 6,0 & $5,2-6,8$ & $191 / 3294$ & 5,8 & $5,0-6,6$ \\
\hline Amputación & 0 & 0,0 & $0,0-0,0$ & 25/3 255 & 0,8 & $0,5-1,1$ & $26 / 3420$ & 0,8 & $0,5-1,1$ \\
\hline Cardiovasculares & $13 / 145$ & 9,0 & $4,3-13,6$ & $788 / 3409$ & 23,1 & $21,7-24,5$ & $805 / 3583$ & 22,5 & $21,1-23,8$ \\
\hline
\end{tabular}

a En los totales se consideraron los datos de los 29 pacientes de los que no se logró conocer el tipo de diabetes.

b $f / n$ : frecuencia de la complicación/número de historias clínicas que tenían ese dato.

c IC95\%: intervalo de confianza de $95 \%$. 
sados en evaluaciones de la calidad de la atención con parámetros que permitan reducir las complicaciones. Estos programas permiten identificar los factores que influyen en el control y el tratamiento e implementar medidas destinadas a su optimización.

La proporción de pacientes con diabetes tipo 2 en la muestra (95,1\%) es mayor que la encontrada en los informes de los programas de atención a diabéticos (80-90\%) (15). Esto se debe probablemente al esquema de atención de salud — que se centra en el adulto- - y a que la atención de los niños y las niñas con diabetes está en manos de los endocrinólogos pediatras. En general, en la mayoría de los informes, la proporción de mujeres es ligeramente mayor que la de hombres, principalmente en la diabetes tipo 2 (16).

La práctica de ejercicios, que complementa la terapia farmacológica y permite un mejor control de los demás factores de riesgo, fue menos frecuente en la población estudiada $(56,9 \%)$ que la observada en pacientes estadounidenses $(69,0 \%)$ (17). Se debe hacer un mayor énfasis en promover la práctica sistemática de ejercicios físicos y proporcionar las condiciones adecuadas para que los pacientes puedan realizarlos. El consumo de grasas encontrado en el presente estudio $(24,8 \%)$ fue mucho menor que el encontrado en ese estudio estadounidense $(61,0 \%)$ (17). Es bien conocida la influencia negativa de la obesidad sobre el control metabólico de las personas $(17,18)$, por lo que se debe trabajar para reducir el IMC promedio general encontrado de $28,0 \mathrm{~kg} / \mathrm{m}^{2}$, especialmente en los diabéticos tipo 2 , ya que $74,3 \%$ de ellos presentaron IMC $>25 \mathrm{~kg} / \mathrm{m}^{2}$.

Debido a que la diabetes se considera un equivalente coronario $(4,19)$, las medidas para reducir los factores de riesgo asociados con la diabetes deben constituir una parte importante del tratamiento de esta enfermedad, especialmente para controlar la HTA y la dislipidemia. Esto contribuiría a reducir considerablemente las complicaciones cardiovasculares y renales en estos pacientes (20-25). Por lo tanto, las metas terapéuticas que deben al- canzar los pacientes con diabetes están por debajo de los valores considerados normales para la población general. El subdiagnóstico de la dislipidemia en las historias clínicas fue de 30,5\%; si se tienen en cuenta los criterios del Tercer Panel de Tratamiento del Adulto (ATP III) del Programa Nacional de Educación en Colesterol (NCEP) de los Institutos Nacionales de Salud de los Estados Unidos, la proporción de pacientes con dislipidemia fue de $98,4 \%$. Estos resultados respaldan las recomendaciones del Estudio Colaborativo de Atorvastatina en Diabetes (CARDS) en el sentido de que se deben prescribir medicamentos hipolipemiantes a todos los pacientes con diabetes tipo 2 . Según los resultados de la Encuesta Nacional sobre Salud y Nutrición (NHANES), 58,0\% de los estadounidenses con diabetes tenían valores de colesterol por encima de $200 \mathrm{mg} / \mathrm{dL}$ en 1999-2000 (26), mientras que en el presente estudio la proporción de pacientes con colesterol total por encima de $180 \mathrm{mg} / \mathrm{dL}$ fue de 53,2\%. Otros estudios realizados en poblaciones diferentes encontraron entre $22 \%$ y $26 \%$ de los pacientes diabéticos con dislipidemia (27), aunque con niveles umbrales mayores que los utilizados en este estudio.

El cumplimiento de las metas de control del colesterol de LDL, triglicéridos y colesterol de HDL fue insuficiente $(14,2 \%, 36,9 \%$ y $47,4 \%$, respectivamente) y refleja el deficiente cumplimiento de las recomendaciones del ATP III de usar medicamentos hipolipemiantes. En el presente estudio, solo $48,0 \%$ de los pacientes con diabetes tomaba este tipo de medicamentos. Este valor es similar al encontrado en diferentes poblaciones estadounidenses (entre $30 \%$ y $54 \%$ ) y se refleja en el bajo porcentaje $(14,2 \%)$ de pacientes con valores de colesterol de LDL por debajo de $100 \mathrm{mg} / \mathrm{dL}$ en ese país $(26,28,29)$.

El tratamiento de la diabetes ha cambiado radicalmente en los últimos años gracias al desarrollo de nuevos medicamentos y a la posibilidad de combinar medicamentos para alcanzar las metas de control propuestas. El porcentaje de pacientes con diabetes tipo 2 que utilizaba insulina fue de
$19,6 \%$, muy bajo si se compara con la proporción de 25-30\% informado en otros países $(27,30,31)$. Esto, que puede deberse al rechazo del médico o del paciente, conduce a un inadecuado control de la diabetes y es una de las causas de la aparición de complicaciones. No obstante, la elevada proporción de pacientes que combinan dos medicamentos o más $(37,2 \%)$ en la población estudiada puede reflejar un intento de controlar mejor la enfermedad. Solo $42,8 \%$ del total de pacientes estudiados presentaba valores de glucohemoglobina por debajo de $7,0 \%$, similar a las cifras cercanas a $40 \%$ encontradas por otros autores en otros países $(15,29,32)$.

La prevalencia general de HTA fue de $68,8 \%$ y su frecuencia fue mayor en los pacientes con diabetes tipo 2 $(71,1 \%)$-igual a la encontrada en el NHANES III (33) y superior al 58,0\% informada en Finlandia (34) — que en los de tipo 1, aunque esta fue similar a la encontrada en varios países europeos $(24 \%)$ en este tipo de pacientes (35). En general, el control de la tensión arterial fue insuficiente: solo $46,3 \%$ de los pacientes tenía la tensión arterial sistólica dentro de los límites recomendados y 36,8\% tenía la tensión arterial diastólica bajo control. Esta situación no refleja el amplio uso de medicamentos en $97,1 \%$ de los pacientes con HTA, de los cuales 58,2\% usaban combinaciones de medicamentos. Estos resultados son similares a los encontrados en otras poblaciones, en las que solo 30-50\% de los pacientes lograban mantener su tensión arterial dentro de los límites establecidos $(26,27,29,36)$.

La única forma de incidir favorablemente en la evolución de esta enfermedad es mejorar el grado de cumplimiento de las metas, tanto clínicas como de laboratorio. Sin embargo, estos resultados demuestran que el cumplimiento de las metas de control metabólico fue insatisfactorio y varió entre $14,2 \%$ y $47,4 \%$, similar a lo encontrado en otros países (37).

Las complicaciones crónicas de la diabetes constituyen el principal problema en este grupo de pacientes, no solo desde el punto de vista médico sino también del económico. Se calcula 
que alrededor de $70 \%$ del total de los costos del tratamiento de la diabetes corresponde al tratamiento de sus complicaciones (6). En el presente estudio, la prevalencia de las complicaciones varió entre 5,8\% (pie diabético) y $31,8 \%$ (retinopatía y nefropatía). La prevalencia de microalbuminuria en la población estudiada $(14,1 \%)$ fue más frecuente en los pacientes con diabetes tipo 1 (20,5\%; IC95\%: 12,0 a 28,9\%) que en los de tipo 2 (13,8\%; IC95\%: 12,4 a $15,2 \% ; P=0,019)$, debido probablemente al gran número de omisiones de los valores de microalbuminuria en las historias clínicas. Según los resultados del estudio EURODIAB, la prevalencia de microalbuminuria fue de $30 \%$ en pacientes con diabetes tipo 1, mientras que la de retinopatía fue de $46 \%$ (38). En un estudio realizado en los Estados Unidos se encontró una mayor prevalencia de nefropatía en pacientes de origen hispano (33,4\%; IC95\%: 26,7 a $40,1 \%$ ) y africano (26,5\%; IC95\%: 19,3 a $33,6 \%)$ que en anglosajones (18,2\%; IC95\%: 12,9 a $23,6 \%$ ) (39).

En este estudio, las enfermedades cardiovasculares fueron mucho menos frecuentes en los pacientes con diabetes tipo $1(9,0 \%)$ que en los de tipo 2 $(23,1 \%)$; la enfermedad coronaria fue la más frecuente en los pacientes con diabetes tipo 2 (13,3\%). Los valores de prevalencia de la enfermedad cardiovascular encontrados en el Estudio Epidemiológico de Complicaciones de la Diabetes (EDC) de Pittsburg, Estados Unidos de América, y el Estudio Europeo de Complicaciones de la Diabetes Tipo 1 (EURODIAB) variaron entre $7,4 \%$ y $8,6 \%(40,41)$, similares a los de la población colombiana estu- diada aquí. Las referencias encontradas sobre la prevalencia de la enfermedad cardiovascular en diabéticos tipo 2 varían entre $10,7 \%$ y 30,0\% $(15,42)$.

Uno de los principales problemas encontrados en este y en otros estudios basados en historias clínicas es la falta de datos. Entre los objetivos del presente estudio estaba determinar la frecuencia de la ausencia de datos en las historias clínicas, ya fuera por deficiencias en las políticas de las instituciones o por errores de los integrantes del equipo de salud. En Colombia no se han establecido algoritmos específicos de evaluación integral de los factores que intervienen en la atención al paciente diabético y se cumplen las guías internacionales y sus recomendaciones para el seguimiento $(2,14)$. Se debe resaltar que los datos de seguimiento de la nefropatía y de la retinopatía —dos complicaciones cuya atención resulta muy costosa- fueron los que con mayor frecuencia se omitieron en las historias clínicas, seguidas de la determinación del colesterol de LDL y la dislipidemia. Diferentes publicaciones muestran datos similares $(30,43,44)$.

Una limitación del presente estudio fue la mayor proporción de participantes en las IPS Comfama y Punto Salud, lo que puede deberse a que estas instituciones poseen varios centros de atención y en total atienden a una mayor población. Además, en estas instituciones funcionaban programas de diabetes que cumplían con los criterios de inclusión de este estudio y participaron en él de forma masiva.

Los resultados obtenidos confirman la fuerte asociación entre la diabetes mellitus y la hipertensión, la obesidad y la dislipidemia y que estos factores de riesgo se encuentran insuficientemente controlados en la población estudiada. Solo un porcentaje muy pequeño de pacientes cumplió las metas de tratamiento, por lo que se necesita mejorar los sistemas que conducen a su cumplimiento para poder reducir los riesgos de complicaciones derivadas de la diabetes. Se encontró una baja frecuencia de las tres complicaciones crónicas que conducen a un peor pronóstico y elevan el costo de la atención de salud en los diabéticos: la nefropatía, la retinopatía y la dislipidemia. Se deben poner en práctica medidas de estandarización de las historias clínicas y realizar evaluaciones periódicas de su calidad para reducir el gran subregistro de datos encontrado en ellas.

Agradecimientos. Se agradece a Susalud EPS por el financiamiento brindado a este estudio en Comfama, Punto Salud y el Instituto del Tórax. El grupo de investigación estuvo integrado por Ángela Aguirre, Jesús Bolívar, Alejandra Botero, Alexandra Giraldo, Alexandra Mantilla, Sara Morantes, Carolina Ospina, Luisa Romero, Jorge Ruiz, Cyndi Saldarriaga, Jesús E. Sánchez, Alejandra Tamayo, Marcela Vargas, Alejandro Gallego y Alejandra Zuluaga, todos estudiantes de la Facultad de Medicina de la Universidad de Antioquia; Isabel Duvaltier, de COMFENALCO; John Jairo Orozco, de la Universidad Nacional; Beatriz Mejía, de Coomeva Conquistadores; y Luz Marina Restrepo y Rafael Rodríguez, del Instituto del Tórax.

\section{REFERENCIAS}

1. Buse JB, Polonsky KS, Burant CF. Type 2 diabetes mellitus. En: Larsen PR, Kronenberg HM, Melmed S, Polonsky KS, eds. Williams textbook of endocrinology. 10 ed. Philadelphia: Saunders; 2003. Pp. 1427-84.

2. Aschner P. Guías ALAD de diagnóstico, control y tratamiento de la diabetes mellitus tipo 2. Rev Asoc Latinoam Diabetes. 2000;8(Suppl 1):1-168.
3. Wild S, Roglic R, Green A, Sicree R, King H. Global prevalence of diabetes. Estimates for the year 2000 and projections for 2030. Diabetes Care. 2004;27:1047-53.

4. Expert Panel on Detection, Evaluation, and Treatment of High Blood Cholesterol in Adults. Executive summary of the Third Report of the National Cholesterol Education Program (NCEP) Expert Panel on Detection,
Evaluation, and Treatment of High Blood Cholesterol in Adults (Adult Treatment Panel III). J Am Med Assoc. 2001;285:2486-97.

5. Harris MI. Diabetes in America: epidemiology and scope of the problem. Diabetes Care. 1998;21(Suppl 3):C11-4.

6. American Diabetes Association. Economic costs of diabetes in the U.S. in 2002. Diabetes Care. 2003;26:917-32. 
7. Barcelo A, Aedo C, Rajpathak S, Robles S. The cost of diabetes in Latin America and the Caribbean. Bull World Heath Organ. 2003;81:19-27.

8. Jonsson B. The economic impact of diabetes. Diabetes Care. 1998;(Suppl 3):C7-10.

9. The Diabetes Control and Complications Trial Research Group. The effect of intensive treatment of diabetes on the development and progression of long-term complications in insulin-dependent diabetes mellitus. N Engl J Med. 1993;329:977-86.

10. UK Prospective Diabetes Study (UKPDS) Group. Intensive blood-glucose control with sulphonylureas or insulin compared with conventional treatment and risk of complications in patients with type 2 diabetes (UKPDS 33). Lancet. 1998;352:837-53.

11. Ohkubo Y, Kishikawa H, Araki E, Miyata T, Isami S, Motoyoshi S. Intensive insulin therapy prevents the progression of diabetic microvascular complications in Japanese patients with non-insulin dependent diabetes mellitus: a randomized, prospective 6 year study. Diabetes Res Clin Pract. 1995;28: 103-17.

12. Alba Mendoza O. Complicaciones crónicas de la diabetes mellitus tipo 2: prevalencia en pacientes usuarios del ISS [tesis de grado]. Santa Fe de Bogotá: Universidad Colegio Mayor de Nuestra Señora del Rosario; 1998.

13. Celis D, Villegas A. Prevalencia de complicaciones crónicas en los pacientes diabéticos del CAA Central ISS [tesis de grado]. Medellín: Universidad Pontificia Bolivariana, Facultad de Medicina; 1997.

14. American Diabetes Association. Standards of medical care in diabetes. Diabetes Care. 2004; 27:S15-35

15. Färnkvist LM, Lundman BM. Outcomes of diabetes care: a population-based study. Int J Qual Health Care. 2003;15:301-7.

16. Gale EAM, Gillespie KM. Diabetes and gender. Diabetologia. 2001;44:3-5.

17. Nelson KM, Reiber G, Boyko EJ. Diet and exercise among adults with type 2 diabetes: findings from the Third National Health and Nutrition Examination Survey (NHANES III). Diabetes Care. 2002;25:1722-8.

18. Okosun IS, Dever GE. Abdominal obesity and ethnic differences in diabetes awareness, treatment, and glycemic control. Obes Res. 2002;10:1241-50.

19. Grundy SM, Cleeman JI, Bairey CN, Brewer HB, Clarck LT, Hunninghake DB, et al. Implications of recent clinical trials for the national cholesterol education program. Adult Treatment Panel III guidelines. Circulation. 2004; 110:227-39.

20. Brenner BM, Cooper ME, De Zeeuw D, Keane WK, Micht WE, Parving HH, et al. Effects of losartan on renal and cardiovascular outcomes in patients with type 2 diabetes and nephropathy. N Engl J Med. 2001;345:851-60.

21. Lewis EJ, Hunsicker LG, Clarke WR, Berl T, Pohl MA, Lewis JB, et al. Renoprotective effect of the angiotensin-receptor antagonist irbesartan in patients with nephropathy due to type 2 diabetes. N Eng J Med. 2001;345: 851-60.

22. Pahor M, Psaty BM, Alderman HM, Applegat WB, Williamson JD, Furberg CD. Therapeutic benefits of ACE inhibitors and others antihypertensive drugs in patients with type 2 diabetes. Diabetes Care. 2000;23:888-92.

23. Pyorala K, Pederson TR, Kjekshus J, Faergeman O, Olsson AG. Thorgeirsson G. Cholesterol lowering with simvastatin improves prognosis of diabetic patients with coronary heart disease: a subgroup analysis of the Scandinavian Simvastatin Survival Study (4S). Diabetes Care. 1997;20:614-20.

24. Colhoun HM, Betteridge DJ, Durrington PN, Hitman GA, Neil HA, Livingtone SJ, et al. Primary prevention of cardiovascular disease with atorvastatin in type 2 diabetes in the Collaborative Atorvastatin Diabetes Study (CARDS): multicentre randomised placebocontrolled trial. Lancet. 2004;364:685-9.

25. UK Prospective Diabetes Study Group. Tight blood pressure control and risk of macrovascular and microvascular complications in type 2 diabetes: UKPDS 38. Br Med J. 1998; 317:703-13.

26. Saydah SH, Fradkin J, Cowie CC. Poor control of risk factors for vascular disease among adults with previously diagnosed diabetes. J Am Med Assoc. 2004;291:335-42.

27. Berardis G, Pellegrini F, Franciosi M, Belfiglio M, Di Nardo B, Greenfield S, et al. Quality of care and outcomes in type 2 diabetic patients. A comparison between general practice and diabetes clinics. Diabetes Care. 2004;27: 398-406.

28. Camenga D, Gill JM. Treatment of hypercholesterolemia in patients with diabetes at a family practice office. Del Med J. 2003;75: 415-20.

29. Beaton SJ, Nag SS, Gunter MJ, Gleeson JM, Sajjan SS, Alexander CM. Adequacy of glycemic, lipid, and blood pressure management for patients with diabetes in a managed care setting. Diabetes Care. 2004;27:694-8.

30. Smith NL, Heckbert SR, Bittner VA, Savage PJ, Barzilay JI, Dobs AS, et al. Antidiabetic treatment trends in a cohort of elderly people with diabetes. The Cardiovascular Health Study, 1989-1997. Diabetes Care. 1999;22: 736-42.

31. Wysowski DK, Armstrong G, Governale L. Rapid increase in the use of oral antidiabetic drugs in the United States, 1990-2001. Diabetes Care. 2003;26:1852-5.

32. Shorr RI, Franse LV, Resnick HE, Di Bari M, Johnson KC, Pahor M. Glycemic control of older adults with type 2 diabetes: findings from the Third National Health and Nutrition Examination Survey, 1988-1994. J Am Geriatr Soc. 2000;48:264-7.

33. Geiss LS, Rolka DB, Engelgau MM. Elevated blood pressure among U.S. adults with diabetes, 1988-1994. Am J Prev Med. 2002;22: 42-8.

34. Hanninen JA, Takala JK, Keinanen-Kiukaanniemi SM. Blood pressure control in subjects with type 2 diabetes. J Hum Hypertens. 2000; 14:111-5.

35. Collado-Mesa F, Colhoun HM, Stevens LK, Boavida J, Ferriss JB, Karamanos B, et al. Prevalence and management of hypertension in type 1 diabetes mellitus in Europe: The EURODIAB IDDM Complications Study. Diabet Med. 1999;16:41-8.

36. Schaars CF, Denig P, Kasje WN, Stewart RE, Wolffenbuttel BHR, Haaijer-Ruskamp FM. Physician, organizational, and patient factors associated with suboptimal blood pressure management in type 2 diabetic patients in primary care. Diabetes Care. 2004;27:123-8.

37. Putzer GJ, Ramirez AM, Sneed K, Brownlee HJ, Roetzheim RG, Campbell RJ. Prevalence of patients with type 2 diabetes mellitus reaching the American Diabetes Association's target guidelines in a university primary care setting. South Med J. 2004;97:145-8.

38. Microvascular and acute complications in IDDM patients: The EURODIAB IDDM Complications Study. Diabetologia. 1994;37:278-85.

39. Harris MI, Klein R, Cowie CC, Rowland M, Byrd-Holt DD. Is the risk of diabetic retinopathy greater in non-Hispanic blacks and Mexican Americans than in non-Hispanic whites with type 2 diabetes? A U.S. population study. Diabetes Care. 1998;21:1230-5.

40. Orchard TJ, Stevens LK, Forrest KY, Fuller JH. Cardiovascular disease in insulin dependent diabetes mellitus: similar rates but different risk factors in the U.S. compared with Europe. Int J Epidemiol. 1998;27:976-83.

41. Koivisto VA, Stevens LK, Mattock M, Ebeling $\mathrm{P}$, Muggeo M, Stephenson J, et al. Cardiovascular disease and its risk factors in IDDM in Europe. EURODIAB IDDM Complications Study Group. Diabetes Care. 1996;19:689-97.

42. Zafra Mezcua JA, Méndez Segovia JC, Novalbos Ruiz JP, Costa Alonso MJ, Failde Martínez I. Chronic complications in patients with type 2 diabetes mellitus cared for at a health center. Aten Prim. 2000;25:529-35.

43. Tapp RJ, Zimmet PZ, Harper CA, de Courten MP, Balkau B, McCarty DJ, et al. Diabetes care in an Australian population: frequency of screening examinations for eye and foot complications of diabetes. Diabetes Care. 2004;27: 688-93.

44. Saaddine JB, Engelgau MM, Beckles GL, Gregg EW, Thompson TJ, Narayan KM. A diabetes report card for the United States: quality of care in the 1990s. Ann Intern Med. 2002; 136:565-74.

Manuscrito recibido el 9 de febrero de 2006. Aceptado para publicación, tras revisión, el 6 de julio de 2006. 
ABSTRACT Objectives. To identify the principal sociodemographic, clinical, and behavioral characteristics of patients participating in diabetic care programs in the city of Medellín, Colombia, and to evaluate progress toward the goal of metabolic control and dia-

Controlling diabetes mellitus and its complications in Medellín, Colombia, 2001-2003
Methods. A cross-sectional, descriptive study was done, analyzing the clinical records of patients who had been participating for at least 6 months in the diabetic care programs of nine health care centers in Medellín. The study period ran from January 2001 to December 2003. The following data were collected: sociodemographic (age and sex), clinical (time since disease onset, diabetes type and treatment, and concurrent illnesses and their treatment), lifestyle habits (exercise routine, smoking, and sugar and fat consumption), chronic complications, and laboratory test results from the prior year. Questionable or missing data were categorized as unavailable.

Results. Of the 3583 clinical histories evaluated, we were able to confirm the form of diabetes for 3554 patients. Of those $3554,95.1 \%$ had type 2 diabetes. Overall, $56.9 \%$ (95\% confidence interval (95\% CI): $55.2 \%$ to $58.6 \%$ ) of the patients exercised regularly, $15.1 \%$ (95\% CI: $13.9 \%$ to $16.3 \%$ ) were current smokers, $17.7 \%$ (95\% CI: $16.4 \%$ to $19.0 \%$ ) were former smokers, and $67.2 \%$ (95\% CI: $65.6 \%$ to $68.8 \%$ ) had never smoked. In all, $21.0 \%$ (95\% CI: $19.6 \%$ to $22.5 \%$ ) of the patients consumed sugar regularly, while $24.8 \%$ (95\% CI: $23.3 \%$ to $26.3 \%$ ) consumed fats and only $19.5 \%$ (95\% CI: $17.8 \%$ to $21.3 \%$ ) were self-monitoring their glucose levels at least weekly. Among the type 2 diabetics, $68.8 \%$ had high blood pressure, and $98.2 \%$ had dyslipidemia. The average body mass index was $28.0 \mathrm{~kg} / \mathrm{m}^{2}$ (95\% CI: 27.8 to 28.2$)$. The average number of medications that the patients were taking to treat type 2 diabetes was 1.3; to treat high blood pressure, 1.9; and to treat dyslipidemia, 0.6. There were no data on low-density lipoprotein (LDL) cholesterol in $44.8 \%$ of the clinical records, and no data on highdensity lipoprotein (HDL) cholesterol in $16.4 \%$ of the records. Among the complications, the most common were ocular ones $(31.8 \%$; $95 \%$ CI: $30.1 \%$ to $33.5 \%)$, followed by renal ones $(25.9 \%$; $95 \%$ CI: $24.4 \%$ to $27.5 \%)$, and cardiovascular ones $(22.5 \%$; 95\% CI: $21.1 \%$ to $23.8 \%$ ). Cholesterol control achievement was inadequate for LDL (reached by only $14.2 \%$ of the patients), for triglycerides (36.9\% of patients), and HDL (47.4\% of patients).

Conclusions. Among the study population in Medellín, the three chronic complications with the greatest impact on prognosis and health care costs of the diabetics were nephropathy, retinopathy, and dyslipidemia. The noticeable amount of unavailable data in the clinical records could be greatly reduced by standardizing the clinical record forms and by periodic quality checks of the records themselves.

Key words Diabetes mellitus, diabetes complications, Colombia. 